Pel l et charge exchange hel i um measur ement using neutral parti cle anal yzer in I arge hel i cal devi ce

\begin{tabular}{|l|l|}
\hline $\begin{array}{l}\text { j our nal or } \\
\text { publ i cat i on } \mathrm{t} \text { i t l e }\end{array}$ & Revi ew of Sci ent i f i c I nst r ument s \\
\hline vol une & Vol . 79 \\
\hline page r ange & 10E518 1 - 10E518 4 \\
\hline year & 2008 10 - 01 \\
\hline URL & ht t p: //hdl . handl e. net /10655/3811 \\
\hline
\end{tabular}




\title{
Pellet charge exchange helium measurement using neutral particle analyzer in large helical device ${ }^{a)}$
}

\author{
T. Ozaki, ${ }^{1}$ P. Goncharov,${ }^{1}$ E. Veshchev,${ }^{1}$ N. Tamura, ${ }^{1}$ S. Sudo, ${ }^{1}$ T. Seki, ${ }^{1}$ H. Kasahara, ${ }^{1}$ \\ Y. Takase, ${ }^{2}$ and T. Ohsako \\ ${ }^{1}$ High Energy Particle Group, Wave Heating Group and LHD Experimental Group, National Institute \\ for Fusion Science, Toki, Gifu 509-5292, Japan \\ ${ }^{2}$ Department of Complexity Science and Engineering, Graduate School of Frontier Sciences, The University \\ of Tokyo, Kashiwa, Chiba 277-8561, Japan
}

(Presented 12 May 2008; received 7 May 2008; accepted 29 July 2008;

published online 31 October 2008)

\begin{abstract}
It is very important to investigate the confinement of $\alpha$ particles, which will be produced by nuclear reactions in ITER and fusion reactors. The pellet charge exchange (PCX) measurement is one of the most powerful methods because it can directly provide the profile of the $\alpha$ particle energy spectra in a plasma. In the large helical device, PCX using tracer encapsulated solid pellet (TESPEL) has been tried in many hydrogen and helium plasmas, including helium accelerated by using the cyclotron resonance heating. In the PCX, we use the compact neutral particle analyzer without simultaneous mass separation ability. The helium particle measurement can be achieved by the application of voltage in the condenser plate. The scattering of hydrogen particle is carefully considered during the estimation of the helium amount. The radial helium profiles can also be obtained by comparing four TESPEL injection shots with/without higher harmonic fast wave heating and at applied plate voltages for $\mathrm{He}$ or $\mathrm{H}$, respectively. () 2008 American Institute of Physics. [DOI: 10.1063/1.2978191]
\end{abstract}

\section{INTRODUCTION}

High-energy particles including $\alpha$ particles are lost not only by charge exchange but also by magnetohydrodynamics (MHD) instabilities such as the Alfvén eigenmode in ITER or a fusion reactor. ${ }^{1}$ These particles can damage the plasma wall in addition to creating poor plasma confinement. Therefore, a suitable method for measuring $\alpha$ particle/helium ion distribution is needed. In the large helical device (LHD), ${ }^{2}$ it is easily possible to do a simulation experiment of $\alpha$ particle heating by using ion cyclotron resonance heating (ICH). ${ }^{3}$

However, it is very difficult to use the passive charge exchange neutral particle method for helium ions. Helium ions are almost fully ionized except near the peripheral region. Few helium atoms escape from plasmas by the double charge exchange reaction between the background helium neutral and the fully ionized helium ion, the cross section of which is too small. A portion of the reionized helium ions, which is generated in a stripping cell (or foil), cannot reach the detector because the scattering between the gas (or foil) target and the helium atom is too large. Therefore the detection efficiency for the helium is too small. A pellet charge exchange (PCX) measurement is well known as one of the most powerful methods to obtain the spatial resolved $\alpha$ particle or helium energy spectrum. ${ }^{4,5}$ The method can be utilized for not only $\alpha$ particle measurement but also the radial energy profile of other energetic particle in various plasmas.

\footnotetext{
a) Contributed paper, published as part of the Proceedings of the 17th Topical Conference on High-Temperature Plasma Diagnostics, Albuquerque, New Mexico, May 2008.
}

Here we make clear the feasibility of PCX for the measurement of the radial profile of the high-energy particle spectrum through the experiments on neutral beam injection (NBI) plasmas in LHD. In order to observe the helium ion, we change the plate voltage of the particle analyzer mainly during the higher harmonic fast wave (HHFW) heating experiments.

\section{PELLET CHARGE EXCHANGE}

High-energy neutral particles, which are produced by the charge exchange between the injected tracer encapsulated solid pellet ${ }^{6}$ (TESPEL) and the energetic ions, are observed by PCX. The TESPEL is ablated and produces an ablation cloud with several layers of different charge states around the traveling pellet in the plasma. A portion of the energetic ions passing through the pellet ablation cloud becomes neutrals due to the charge exchange reaction and escape from the confinement of magnetic field in the plasma. Helium measurements in the plasma are possible because double charge exchange or two sequential single charge exchange events are possible if a high- $Z$ material such as carbon or its compound is used. The neutralization factor is needed in order to obtain the energy spectrum in the plasma. It is determined by the ratio of the recombination to the ionization. ${ }^{7}$

One of the advantages of PCX is the spatial information. When the compact neutral particle analyzer ${ }^{8}$ (CNPA) for measurement of the charge exchanged particle is installed just behind the TESPEL trajectory, the time trace of the signal can be converted to the information as a function of the pellet position. The typical pellet velocity of $400 \mathrm{~m} / \mathrm{s}$ gives a 


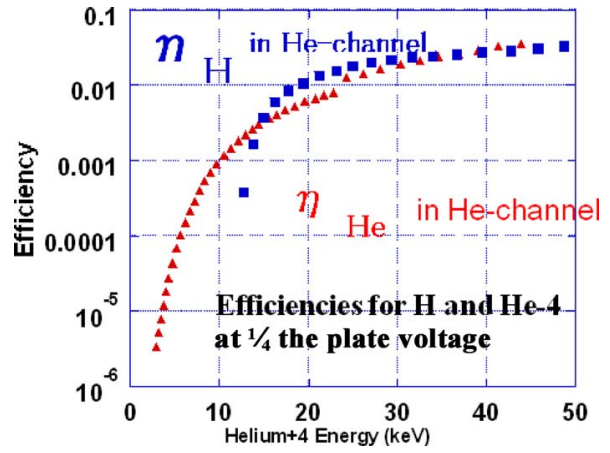

FIG. 1. (Color online) Efficiencies for He-4 and scattered hydrogen at the plate voltage of 1/4 times of hydrogen. Even if the plate voltage is adjusted to the He channel, the scattered hydrogen is still observed.

spatial resolution of $4 \mathrm{~cm}$ for the sampling time of $0.1 \mathrm{~ms}$. This full trajectory of the pellet in the plasma should be in the viewing cone of the CNPA by minimizing the angle between the sight line and the trajectory.

If the plate voltage is changed, a different mass such as helium can be observed in principle. We calibrate the analyzer for helium by the following procedure.

(a) Compare the simulation model ${ }^{9}$ including an accurate orbit calculation with the experimentally calibrated value in hydrogen.

(b) Comparison in (a) is nearly perfect. Therefore, we believe that the simulation model is accurate and we calculate the efficiency in helium and scattered hydrogen when the plate voltage is set to be $1 / 4$ times that for hydrogen.

(c) The calculated efficiencies for helium and the scattered hydrogen are shown in Fig. 1.

\section{EXPERIMENTAL RESULTS (HYDROGEN IN NBI PLASMAS)}

To confirm the extended feasibility of PCX, we tried to measure the radial profile of the high-energy particle spectra by perpendicular injection of TESPEL into NBI plasmas. PCX has been done in NBI plasmas with different magnetic axes in order to compare those spectra. It is known that the inner shift of the magnetic axis provides better confinement of energetic particles in helical devices from computer simulation results. 10

In the experiment, TESPEL is injected to the plasma produced by two tangential NBIs, NBI No. 1 (counterinjection against the magnetic field) and NBI No. 2 (coinjection), and the perpendicular NBI No. 4. Therefore, the initial angular distribution is roughly uniform. Figure 2 shows the radial energy spectra measured by the CNPA. The flux intensity is colored (scale is shown in color chart on the right) so we can easily compare those discrepancies. TESPEL reaches $\rho=0.6$, where $\rho$ is the normalized radius $r / a$ ( $a$ is the minor radius). The time behavior of each energy flux during TESPEL injection can be obtained. The radial energy profile can be obtained by comparing the pellet traveling time with the signal as shown. To obtain the accurate energy spectra, the signal intensity should be divided by the neutralization (a)

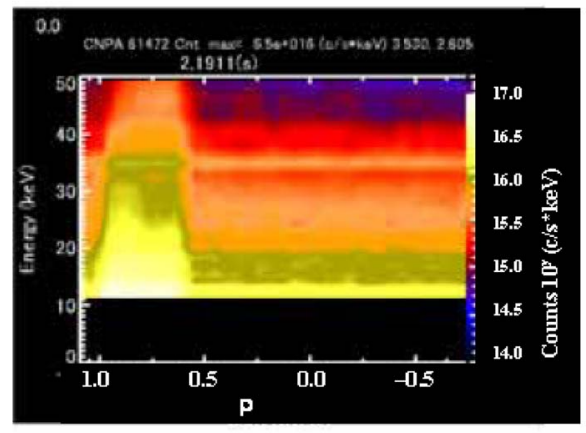

(b)

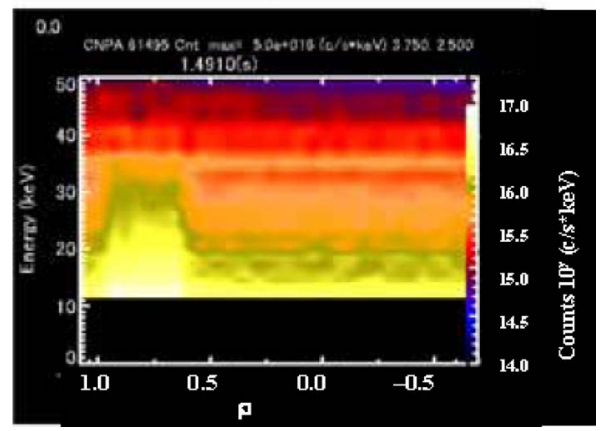

FIG. 2. (Color online) PCX signals. The signals show the radial neutral flux profiles. The flux intensity with logarithmic is colored (a) at $R_{\mathrm{ax}}=3.53 \mathrm{~m}$, $B=2.8 \mathrm{~T}$ and (b) at $R_{\mathrm{ax}}=3.75 \mathrm{~m}, B=2.5 \mathrm{~T}$.

probability based on the charge exchange cross sections. Here for simplification, we concentrate on the qualitative discussion. As mentioned above, better confinement of energetic particles can be obtained by the inner shift of the magnetic axis in helical devices because the plasma region overlaps the particle orbit. Therefore, the increase in flux from the PCX can be expected at the inner axis shift. The maximum flux over a wide energy region can be obtained at the inner magnetic axis shift, especially at the plasma edge. We conclude that PCX is a useful technique to observe the radial energy spectrum of the energetic beam injected ions.

\section{EXPERIMENTAL RESULTS (HELIUM IN ICH PLASMA)}

By using ICH with a higher harmonic fast wave, helium ion acceleration can be expected. This heating is utilized mainly for electron heating using Landau damping. ${ }^{11} \mathrm{We}$ choose the suitable combination between the magnetic field and the frequency of the ICH so that there is no ion cyclotron resonance layer for the hydrogen in the plasma core region. One of their combinations realizes the resonance layer for the $\mathrm{He}^{4}$ positioned in the plasma core region.

In the measurement of helium ion by the PCX, hydrogen still gets mixed in the detector because the charge and mass of hydrogen are close to that of helium. However, the accelerated hydrogen ions are few because there is no resonance layer for hydrogen if the combination of the magnetic field of $1.86 \mathrm{~T}$ and the ion cyclotron frequency of $38.47 \mathrm{MHz}$ is 


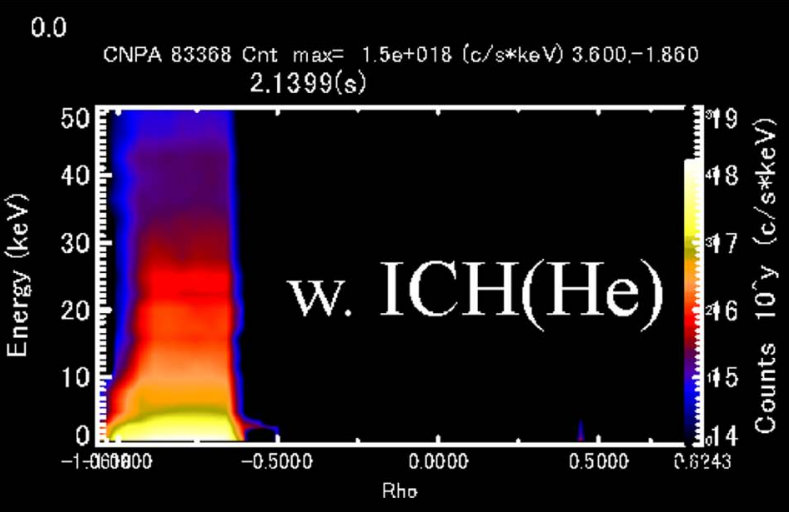

0801311743
0.0

CNPA 83381 Cnt max $=5.6 \theta+017(\mathrm{c} / \mathrm{s} * \mathrm{keV}) 3.600-1.860$ $2.4915(\mathrm{~s})$

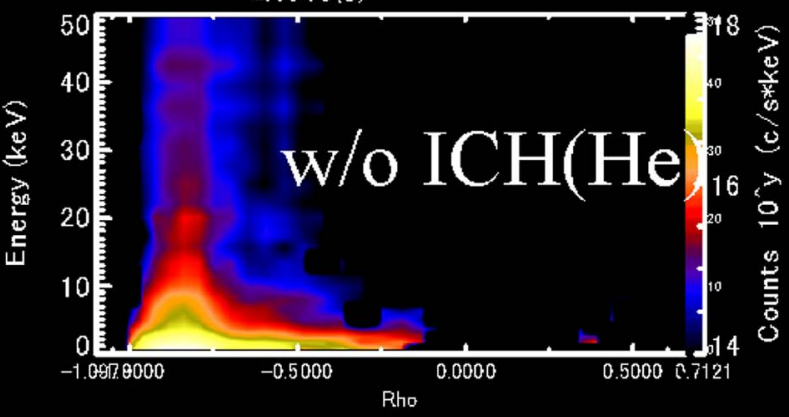

0801311832
0.0

CNPA $83375 \mathrm{Cnt} \max =1.8 \Theta+018(\mathrm{c} / \mathrm{s} * \mathrm{k} \Theta \mathrm{V}) 3.600,-1.860$

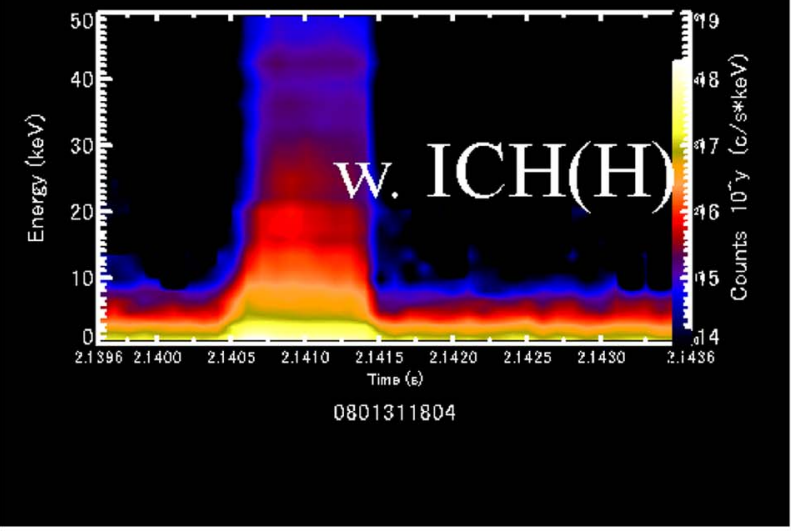

0.0

CNPA $83379 \mathrm{Cnt} \max =2.1 \mathrm{e}+018(\mathrm{c} / \mathrm{s} * \mathrm{k} \Theta \mathrm{V}) 3.600 .-1.860$ $2.4922(s)$

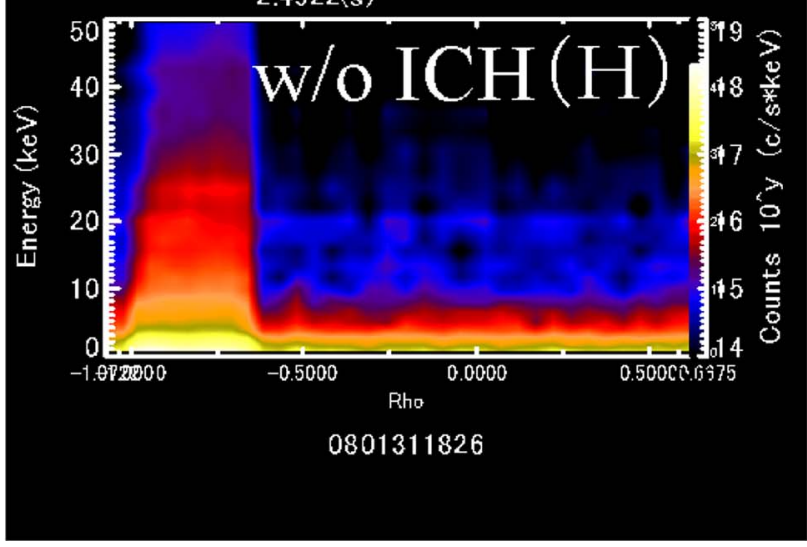

FIG. 3. (Color online) Four PCX signals set on He channel and H channel with/without HHFW heating. To confirm helium acceleration, we compare four different PCX signals.

used. The $\mathrm{He}^{4}$ resonance layer appears at $\rho=1 / 3$ in the third harmonic of the ICH frequency. On the other hand, there are hydrogen resonance layers only at the peripheral region of the plasma.

In the experiment, the plasma is produced by the two tangential NBIs, NBI No. 1 and NBI No. 3. The ICH is intermittently applied. The TESPEL is injected into the plasma after NBI injection in order to minimize the effect of protons generated from NBI. Four similar discharges with TESPEL injection are shown. In two discharges, we measure the helium and hydrogen, respectively. In the next two discharges, the same procedure is performed without ICH.

Figure 3 shows the time histories of the hydrogen and helium energy spectra in four similar shots. The line averaged plasma density of $2 \times 10^{19} \mathrm{~m}^{-3}$ and the central plasma temperature of $2 \mathrm{keV}$ can be observed. To confirm the helium acceleration, we compare the spectra of the helium and hydrogen by using a CNPA with different plate voltages. We must remember that most signals are hydrogen even if we set the plate voltage for helium. TESPELs reach $\rho=0.6$ in all discharges. As mentioned above, the hydrogen component is mixed even if we set the helium channel. Therefore, we use the ratio of $\mathrm{He} / \mathrm{H}$. Figure 4 shows the energy resolved $\mathrm{He} / \mathrm{H}$ ratio profiles with/without $\mathrm{ICH}$. The acceleration can be observed at the wide area between $\rho=0.7-0.85$. We can con-
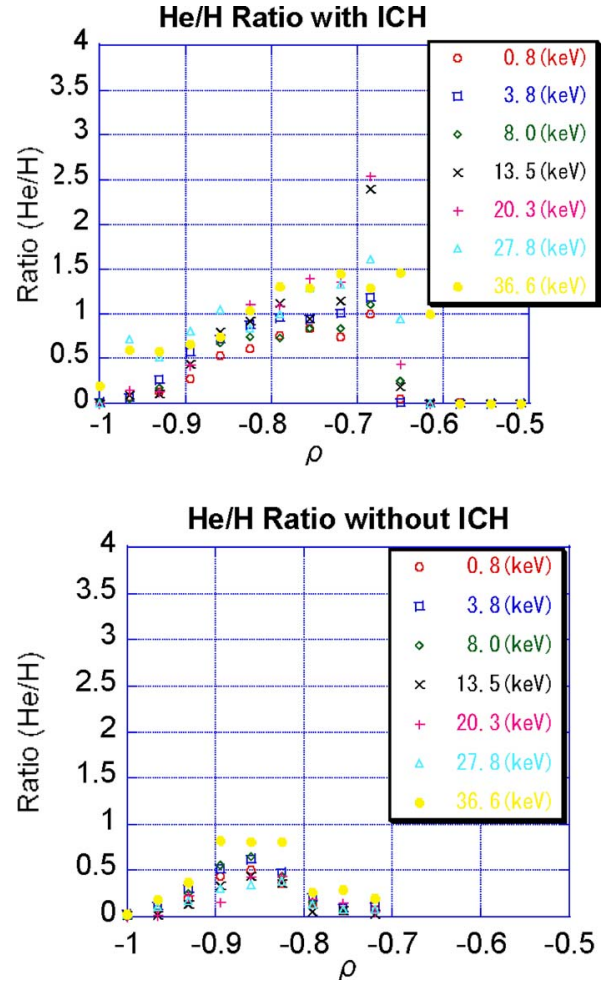

FIG. 4. (Color online) Helium profiles with/without HHFW heating. The higher flux of He can be observed during the HHFW heating. 
firm the acceleration of the helium ion by the HHFW heating from the ratio profiles. This means that PCX is a useful technique for the profile measurement of helium ions or $\alpha$-particles.

\section{SUMMARY}

It is very important to establish the alpha profile measurement because the helium/alpha has a MHD instability in the form of an Alfvén eigenmode. PCX is one of the techniques capable of obtaining the energetic helium particle distribution in a plasma. We demonstrate the feasibility of the energetic hydrogen profile measurements in NBI plasmas. The accelerated helium ion profile created by higher harmonic ICH was also observed by the PCX, where the plate voltage is adjusted to observe He ions.

\section{ACKNOWLEDGMENTS}

This work was performed under NIFS07ULBB509, Grant in Aid Nos. 17540475 and 18035013.

${ }^{1}$ L. Chen, Phys. Plasmas 1, 1519 (1994).

${ }^{2}$ O. Motojima et al., Fusion Eng. Des. 20, 3 (1993).

${ }^{3}$ T. Mutoh et al., Phys. Rev. Lett. 85, 4530 (2000).

${ }^{4}$ R. Fisher et al., Phys. Rev. Lett. 75, 846 (1995).

${ }^{5}$ P. Goncharov et al., Fusion Sci. Technol. 50, 222 (2006).

${ }^{6} \mathrm{~S}$. Sudo et al., Proceedings of the 17th IAEA Fusion Energy Conference, Yokohama, Japan, 1998 (unpublished), Paper No. IAEA-CN-69/EXP1/18, p. 39.

${ }^{7}$ P. Goncharov et al., L14, HTPD2008.

${ }^{8}$ P. Goncharov et al., Rev. Sci. Instrum. 74, 1869 (2003).

${ }^{9} \mathrm{~V}$. Afanasyev, personal communication (2004).

${ }^{10}$ T. Kamimura et al., personal communication (1987).

${ }^{11} \mathrm{H}$. Kasahara et al., Proceedings of the Fifth Asia Plasma Fusion Association, Jeju, Korea, 2005 (unpublished). 